\title{
The waal gene mutation compromised the inhabitation of Enterobacter sp. Ag1 in the mosquito gut environment
}

\author{
Dong $\mathrm{Pei}^{\dagger}$, Jinjin Jiang ${ }^{\dagger}$, Wanqin Yu, Phanidhar Kukutla, Alejandro Uentillie and Jiannong Xu ${ }^{*}$ (i)
}

\begin{abstract}
Background: The mosquito gut harbors a variety of bacteria that are dynamically associated with mosquitoes in various contexts. However, little is known about bacterial factors that affect bacterial inhabitation in the gut microbial community. Enterobacter sp. Ag1 is a predominant Gram negative bacterium in the mosquito midgut.

Methods: In a mutant library that was generated using transposon Tn5-mediated mutagenesis, a mutant was identified, in which the gene waal was disrupted by the Tn5 insertion. The waal encodes $O$ antigen ligase, which is required for the attachment of $\mathrm{O}$ antigen to the outer core oligosaccharide of the lipopolysaccharide (LPS).

Results: The waaL $L^{-}$mutation caused the $O$ antigen repeat missing in the LPS. The normal LPS structure was restored when the mutant was complemented with a plasmid containing waal gene. The waal $L^{-}$mutation did not affect bacterial proliferation in LB culture, the mutant cells grew at a rate the same as the wildtype (wt) cells. However, when waal ${ }^{-}$strain were co-cultured with the wt strain or complemented strain, the mutant cells proliferated with a slower rate, indicating that the mutants were less competitive than wt cells in a community setting. Similarly, in a co-feeding assay, when fluorescently tagged wt strain and waal ${ }^{-}$strain were orally co-introduced into the gut of Anopheles stephensi mosquitoes, the mutant cells were less prevalent in both sugar-fed and blood-fed guts. The data suggest that the mutation compromised the bacterial inhabitation in the gut community. Besides, the mutant was more sensitive to oxidative stress, demonstrated by lower survival rate upon exposure to $20 \mathrm{mM} \mathrm{H}_{2} \mathrm{O}_{2}$.
\end{abstract}

Conclusion: Lack of the $\mathrm{O}$ antigen structure in LPS of Enterobacter compromised the effective growth in co-culture and co-feeding assays. In addition, O-antigen was involved in protection against oxidative stress. The findings suggest that intact LPS is crucial for the bacteria to steadily stay in the gut microbial community.

Keywords: Enterobacter sp. Ag1, Lipopolysaccharide, waaL gene, O antigen ligase, Inhabitation, Oxidative stress, Mosquito gut, Malaria mosquito

\section{Background}

Vector mosquitoes are responsible for the transmission of deadly diseases, such as malaria and Dengue fever, which poses serious threats to human health and severe burdens to socioeconomic development. Mosquito control is one of the effective intervention strategies to interrupt transmission of mosquito-borne diseases. A dynamic microbial community resides in the mosquito gut ecosystem [1-10].

\footnotetext{
* Correspondence: jxu@nmsu.edu

${ }^{\dagger}$ Equal contributors

Biology Department, New Mexico State University, Las Cruces, NM 88003, USA
}

Many efforts have been made in characterizing taxonomic structure of mosquito gut microbiome. The core bacterial inhabitants have been identified in the phyla Proteobacteria, Bacteroidetes, Actinobacteria and Firmicute. Predominant taxa belong to the families Enterobacteriaceae, Acetobacteraceae, Pseudomonadaceae and Flavobacteriaceae $[3,7,11$, 12]. The microbes have significant impacts on various mosquito life traits, such as development, reproduction, fecundity, immunity and vector competence $[6,9,11,13-23]$. For example, Asaia sp. SF2.1 has been shown to be able to accelerate the larval development of Anopheles gambiae when introduced into the aquatic habitats where

\section{Biomed Central}

(C) 2015 Pei et al. Open Access This article is distributed under the terms of the Creative Commons Attribution 4.0 International License (http://creativecommons.org/licenses/by/4.0/), which permits unrestricted use, distribution, and reproduction in any medium, provided you give appropriate credit to the original author(s) and the source, provide a link to the Creative Commons license, and indicate if changes were made. The Creative Commons Public Domain Dedication waiver (http://creativecommons.org/publicdomain/zero/1.0/) applies to the data made available in this article, unless otherwise stated. 
the mosquito larvae grew [17]. On the opposite, the depletion of Asaia by antibiotic rifampicin would cause delay of the larval development in An. stephensi [18]. The presence of native gut bacteria is essential for maintaining an effective basal immunity that determines the susceptibility to malaria in both An. gambiae [20, 22] and An. stephensi [14, 24]. Apparently, a symbiotic relationship between the gut microbiome and the mosquito hosts largely depends on a stably associated core microbial community.

The structure of gut microbial communities varies upon gut conditions of diet types, i.e., sugar meals or blood meals $[7,21]$. The sugar-fed gut is carbohydrate-rich while the blood-fed gut is protein-rich environment. Previously, we have shown that the microbial composition shifts upon diet changes. In the sugar-fed gut, especially before the first blood meal is taken, the taxonomic composition is more complicated than that in the blood-fed gut. After taking blood meal, taxonomic complexity drops with the enrichment of enteric bacteria [7]. In wild population, the gut bacterial composition varies among individuals [22]. So far, most studies have focused on the taxonomic composition and community structural fluctuations in different stages of the life history. However, little is known about what bacterial factors affect bacteria to establish in a complex microbial community in the gut environment.

Bacteria in the genus Enterobacter were often found in the mosquito guts $[2,3,7,16]$, and in the blood-fed gut Enterobacter was one of the enteric bacteria that expanded following blood ingestion [7]. Therefore Enterobacter would be a good representative for studying how bacteria reside in the gut. In an effort to recognize bacterial factors that are involved in the gut inhabitation, we have isolated a strain of Enterobacter sp. from the gut of a mosquito lab colony [25]. In this study, we generated a Tn5 mediated mutant library of Enterobacter sp. and characterized a mutant, waaL $L^{-}$The waaL encodes lipopolysaccharide (LPS) $\mathrm{O}$ antigen ligase. The waaL mutation caused the lack of $\mathrm{O}$ antigen in the LPS and the increased sensitivity to hydrogen peroxide. In addition, the mutant bacteria were compromised in competing with the wild type bacteria in vitro in the co-culture and in vivo in the mosquito gut, suggesting that the $\mathrm{O}$ antigen associated LPS structure is required for the bacteria to reside in a microbial community in the mosquito gut.

\section{Methods}

\section{Bacterial strains and mutagenesis}

Enterobacter sp. Ag1 was originally isolated from the gut of mosquito Anopheles gambiae [25]. A mutant library was generated using EZ-Tn $5^{\mathrm{Tw}}<\mathrm{R} 6 \mathrm{~K} \gamma$ ori $/ \mathrm{KAN}-2>\mathrm{Tnp}$ Transposome kit (Epicentre, WI). First, the Enterobacter cells were grown in Luria-Bertani (LB) broth at $28{ }^{\circ} \mathrm{C}$ to the exponential phase $\left(\mathrm{OD}_{600 \mathrm{~nm}}=0.7\right)$. Cells were harvested by centrifugation at $5000 \times \mathrm{g}$ for $5 \mathrm{~min}$ and washed twice with $100 \mathrm{~mL}$ ice-cold $\mathrm{H}_{2} \mathrm{O}$ and twice $50 \mathrm{~mL}$ ice-cold $10 \%$ glycerol. Cells were finally suspended in $1 \mathrm{~mL}$ of $10 \%$ ice-cold glycerol, and $100 \mu \mathrm{L}$ aliquot were used for electroporation. A mutant library was prepared following manufacturer's instructions. In brief, $1 \mu \mathrm{L}$ of Tnp transposome was added to the electrocompetent cells, and electroporation (5 ms, $12.5 \mathrm{kV}$ ) was performed using an Eporator (Eppendorf, NY). Electroporated cells were immediately recovered by adding $1 \mathrm{~mL}$ of SOC broth and incubated at $28{ }^{\circ} \mathrm{C}$ for $2 \mathrm{~h}$. The transformants were plated on LB with $40 \mu \mathrm{g} / \mathrm{mL}$ kanamycin (KAN) and incubated at $28{ }^{\circ} \mathrm{C}$ overnight. The colonies were further processed for screening genes with a $\operatorname{Tn} 5$ insertion. The defense capability against anti-oxidative stress was speculated to be associated with the expansion of enteric bacteria in the blood-fed mosquito gut [7]. The wt Enterobacter cells were first examined for the sensitivity to paraquat (Sigma-Aldrich), an oxidative stressor. The bacterial growth was measured in the presence of paraquat at a series of concentration $0,2,4$, 6, $8 \mathrm{mM}$ (unpublished data). The growth inhibitory effect was dose dependent. In the presence of $6 \mathrm{mM}$ paraquat, the bacteria were able to grow but with a reduced growth rate (unpublished data). Therefore, $6 \mathrm{mM}$ paraquat was selected for screening mutants. Single transposed colonies from LB-KAN plates were inoculated in the LB broth with both $40 \mu \mathrm{g} / \mathrm{mL}$ KAN and $6 \mathrm{mM}$ paraquat. The $\mathrm{OD}_{600 \mathrm{~nm}}$ was measured hourly for $5 \mathrm{~h}$. The wt cells were used as control. The transformants that had a growth rate slower than the wt were harvested for gene identification.

\section{Identification of disrupted genes}

To identify the genes having a $\operatorname{Tn} 5$ insertion, genomic DNA was isolated from the mutant cells using DNAzol reagent (Invitrogen, NY) and digested with EcoRI (NEB, MA), which has no cutting site in the Tn5. Subsequently, the digested DNA was purified, and the EcoRI ends were ligated with the T4 DNA Ligase (NEB, MA). The ligation was transformed into electrocompetent EC100D pir-116 E. coli (Epicentre, WI) by electroporation, following the manufacturer's instruction. The transformants were recovered on the LB agar plates with $40 \mu \mathrm{g} / \mathrm{mL}$ KAN overnight at $37{ }^{\circ} \mathrm{C}$. Colony PCR was performed to confirm the presence of R6Kyori and KAN-2 using the primers provided in the kit. Plasmids were extracted from positive colonies. The bacterial gene sequences at the junction with $\operatorname{Tn} 5$ were determined by using the forward and reverse Tn5-specific sequencing primers (supplied in the transposome kit). The bacterial sequences were then mapped against the genome sequence of Enterobacter sp. Ag1 [25] to identify where the $\operatorname{Tn} 5$ insertion was located. 


\section{Complemented waal ${ }^{c}$ strain}

The waaL $^{-}$mutant was complemented with a plasmid with the waaL coding sequence. The entire waaL coding sequence was amplified with PCR using forward primer (5' - cagtctagaGATGGCCCTCACATTATTTTTTTC-3') and reverse primer $\left(5^{\prime}\right.$-cagctcgagTTATTTTTTATTCA GTGCTATCAATAACC-3'). The restriction recognition sites Xba I (tctaga) and Xho I (ctcgag) were included in the forward and reverse primers, respectively. The plasmid pGFPuv was purchased from Clontech. The GFP gene in the plasmid was cut out with Xba I and Xho I, and replaced with the waaL PCR products by ligation. The mutant waaL $L^{-}$cells were transformed with the engineered plasmids. The plasmids were extracted from transformants and verified for the presence of waaL by sequencing.

\section{Visualization of lipopolysaccharide on PAGE}

LPS was extracted from the wt, waaL $L^{-}$and complemented $w_{a a L}{ }^{c}$ using a kit (iNtRON Biotechnology, Bulldog Bio, $\mathrm{NH}$ ) following the manufacturer's instruction. The LPS preparations were separated on $12 \%$ PAGE gels, and stained with LPS staining kit (Invitrogen, NY) following the manufacturer's instruction. The LPS was visualized under UV light.

\section{Bacterial tagging}

To be able to visualize bacteria in the mosquito gut, the wt, $w a a L^{-}$and $w a a L^{c}$ bacteria were tagged with green fluorescent protein (GFP) or red fluorescent protein (RFP), respectively. The GFP carrying plasmid, pGFPuv was obtained from Clontech (Cat\#: 632312). The plasmid was a pUC19 derivative pPD16.43 [26], the GFPuv gene was flanked by multiple cloning sites. To make a RFP carrier using the same plasmid, the GFPuv gene was excised from the plasmid using the enzymes $\mathrm{XbaI}$ and Xhol, and replaced with the RFP gene that was excised out from the pTurboRFP-B (Evrogen, Cat\#: FP233). Therefore, the GFP and RFP genes were carried in the same plasmid. The strategy avoided potential issues that different plasmids might cause. The wt and mutant cells were transformed with pGFPuv and pRFP to generate strains wt/GFP, wt/ RFP, waaL $/$ GFP, waaL / RFP, waaL $L^{c} /$ GFP and $w a a L^{c} /$ RFP. Transformed bacteria were screened on plates for three generations, and stably tagged cells were used for the further experiments.

\section{Bacterial growth and co-culture of tagged bacteria}

The cells of the wt, waaL $L^{-}$mutant and complemented waaL $L^{c}$ strains were cultured overnight at $28{ }^{\circ} \mathrm{C}$, and adjusted to $\mathrm{OD}_{600 \mathrm{~nm}}$ of 0.1 in $20 \mathrm{~mL} \mathrm{LB}$ medium, respectively, to initiate the cultures. The $\mathrm{OD}_{600 \mathrm{~nm}}$ of each culture was measured hourly for $6 \mathrm{~h}$. The growth curves were plotted for each strain. The experiments were conducted in triplicates. In co-culture assays, wt (GFP)/waaL $L^{-}$(RFP), $w_{a a L}^{c}$ (RFP)/waaL $(\mathrm{GFP})$, wt (GFP)/waaL ${ }^{c}$ (RFP) were mixed in the ratio of $1: 1$, respectively, and grew in the same conditions as described above. The cultures were conducted in triplicates. A $5 \mu \mathrm{L}$ of co-culture was placed on a glass slide with cover slip and examined with a fluorescent microscope (Nikon) to visualize the ratio of GFP and RFP tagged bacteria. The tagged bacteria were numerated respectively, the ratios of wt (GFP)/waaL $L^{-}$(RFP), waaL $^{c}$ (RFP)/waaL (GFP), and wt (GFP)/waaL ${ }^{c}$ (RFP) were analyzed by one way ANOVA, and Tukey's method was used for post hoc comparison.

\section{Bacterial co-feeding assay}

Anopheles stephensi was used for the study. The gut bacterial load in the newly emerged mosquitoes was very low, largely because the bacteria in the pupal gut are sequestrated during metamorphosis and are egested with meconium after emergence [27-29]. Therefore, we chose to feed bacteria to newly emerged mosquitoes to establish a desired bacterial community with dominance of introduced bacteria. Tagged bacteria were cultured in LB at $28{ }^{\circ} \mathrm{C}$. Cells were collected at exponential phase and washed with $1 \times$ sterile PBS for three times. The combinations, wt/RFP andwaaL /GFP, waaL/RFP and wt/GFP, wt/RFP and $w a a L^{c} / \mathrm{GFP}, w a a L^{-} / \mathrm{RFP}$ and waaL $^{c} /$ GFP, were mixed in a ratio of $1: 1$ at $\mathrm{OD}_{600 \mathrm{~nm}}$ of 1.2 in a $10 \%$ sucrose sugar solution, respectively. The bacterial quantity in the sugar solution was approximately $1 \times 10^{7}$ colony forming unit $(\mathrm{CFU}) / \mathrm{mL}$. The bacteria were introduced into mosquitoes by oral feeding. Newly emerged mosquitoes were fed on tagged bacteria for 2 days. Then, the sugar-fed guts were dissected and observed using a fluorescent microscopy (Nikon) to visualize the bacteria. The remaining mosquitoes were given a blood meal. At $24 \mathrm{~h}$ post blood feeding, the blood-fed guts were dissected to visualize the bacteria.

\section{Mosquito infection with malaria}

Three cohorts of newly emerged An. stephensi were used. One cohort was fed on regular $10 \%$ sucrose solution as control mosquitoes. Second cohort was fed with the wt bacteria and the third cohort was fed with the mutant bacteria for 2 days, respectively. The three cohorts of mosquitoes were fed on the same mice infected with GFP-tagged P.berghei. Then the wt- and mutant-fed mosquitoes were maintained on sterile sugar meals until dissection. At day 7 post infected blood feeding, mosquito guts were dissected and examined under a florescent microscopy to visualize fluorescent oocysts. The infection pattern among three cohorts was compared by the infection rate (prevalence) and the parasite load per gut. Statistically, the difference of prevalence was tested by $\chi^{2}$, and the difference of oocyst number among three cohorts was compared using the 
ANOVA on ranks, and the post hoc was conducted by Dunn's multiple comparison.

\section{Sensitivity to hydrogen peroxide}

To test the sensitivity to $\mathrm{H}_{2} \mathrm{O}_{2}$, the wt and mutant cells were harvested in logarithmic phase. The cells were resuspended in $1 \times$ PBS and adjusted to $\mathrm{OD}_{600 \mathrm{~nm}}$ of 0.2 . The cells were exposed to $20 \mathrm{mM} \mathrm{H}_{2} \mathrm{O}_{2}$ or blank control for $30 \mathrm{~min}$ at $28{ }^{\circ} \mathrm{C}$, respectively, and then plated on a LB plate and incubated at $28{ }^{\circ} \mathrm{C}$ overnight. The number of colonies was counted. The survival rates were calculated as colony counts of $\mathrm{H}_{2} \mathrm{O}_{2}$ exposed cells vs colony counts of blank control cells. The experiments were conducted in triplicates. The average of the survival rates between the wt and mutant were compared and the statistical significance was tested by one-way ANOVA and post hoc comparison was conducted by Donnett's multiple comparison method.

\section{Ethics statement}

This study was carried out under the NMSU and IACUC guidelines for biomedical research.

\section{Results}

The waaL disruption caused the lack of O-antigen in the LPS structure

A mutant library of Enterobacter sp. Ag1 was generated using an EZ-Tn5 mutagenesis kit. A mutant was identified with the disruption of gene waaL by the transposon Tn5 insertion. The waaL gene codes for $\mathrm{O}$ antigen ligase. Structurally, LPS is comprised of three regions: the lipid anchor (lipid A), the inner and outer core oligosaccharide (OS), and the O-antigenic polysaccharide (O-PS) [30]. The $\mathrm{O}$ antigen ligase is responsible for the ligation of $\mathrm{O}$ antigen to the outer core of LPS [31]. The waaL gene is located in a locus harboring a cluster of genes responsible for the LPS core oligosaccharide biosynthesis and assembly [30]. Figure 1a presents the gene cluster in the genome of Enterobacter sp. Ag1 [25], and Fig. 1b depicts the Tn5 insertion of waaL in the mutant.

The $w_{a a L^{-}}$mutation caused a defect in LPS structure. On a PAGE gel, the LPS of the wt strain displayed a typical ladder-like banding pattern with varying lengths of O-PS repeats (Fig. 2, lane 1). The LPS ladder was almost missing in the $w_{a a L^{-}}$(Fig. 2, lane 2). When the $w_{a a L^{-}}$was complemented with a plasmid carrying a full-length of waaL, the O-PS repeats were restored (Fig. 2, lane 3). This was consistent with the function of $\mathrm{O}$-antigen ligase, mediating the attachment of O-PS to the core of LPS. Interestingly, the lack of O-PS appears not to affect growth in vitro. The cultures of three strains, wt, $w a a L^{-}$and $w a a L^{c}$, showed very similar growth curves (Fig. 3).

\section{The $\mathrm{waal}^{-}$was less competitive than wt both in the gut and in the co-culture in vitro}

Enterobacter is one of the predominant inhabitants in the mosquito gut [7]. To test if the lack of O-PS affects the inhabitance of bacteria in the mosquito gut, fluorescently tagged bacteria were used to track their dynamics in the mosquito gut. Preliminary results showed that the number of ingested wt bacteria varied in different individual guts over time. In addition, it was unfeasible to accurately quantify all tagged bacteria in a gut using gut images. Therefore, we developed a co-feeding assay to examine the colonization capability of the $w a a L^{-}$. The bacteria were tagged with GFP and RFP, respectively. These combinations were used for co-feeding study, wt/RFP and $w a a L^{-} / G F P$, $w a a L^{-} /$RFP and wt/GFP, wt/RFP and $w a a L^{c} / G F P$, waaL -/RFP and $w a a L^{c} /$ GFP. Tagged bacteria were mixed in a ratio of 1:1 in sugar meals (Fig. 4A). Newly emerged $A n$. stephensi were fed with tagged bacteria for 2 days. On the day 3 post bacterial feeding, the mosquito guts were examined for the bacterial status. As shown in Fig. 4B, the wt and complemented bacteria steadily populated in the sugar-fed gut, but $w a a L^{-}$bacteria were less seen in the gut. The mosquitoes were then given a blood meal. The gut bacteria were examined at $24 \mathrm{~h}$ post blood feeding. As shown in Fig. 4C, the mutants were much less prevalent than the wt and complemented bacteria in the blood-fed gut. Apparently, in the co-feeding assay, the mutant cells were less competitive than the wt and complemented cells in the gut environment. Since the mutant cells grew normally in LB culture (Fig. 3), we used a co-culture assay to test if the growth would change in the presence of wt cells. Indeed, the mutant cells grew at a slower rate than the wt cells. As shown in Table 1, the ratios of both wt vs $w a a L^{-}$and $w a a L^{c}$ vs $w a a L^{-}$were around 1 in the initial of

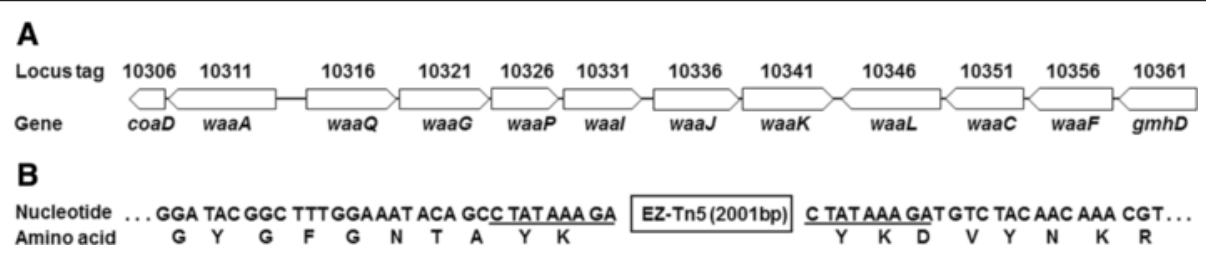

Fig. 1 Genomic location of waaL and Tn5 insertion in waaL. a The genomic region where a cluster of waa genes are located. The locus tag (the prefix A936_was omitted) and gene names were indicated. The waal gene encodes a 402 aa protein. The waal gene was boxed. $\mathbf{b}$ Insertion site of the Ez-Tn5 transposon in the waal gene. The transposon was inserted between the 2nd (A) and 3rd (T) nucleotide of the 298th codon (GAT/D) and generated a 9 bp direct repeat (underlined) flanking the Tn5 insert 


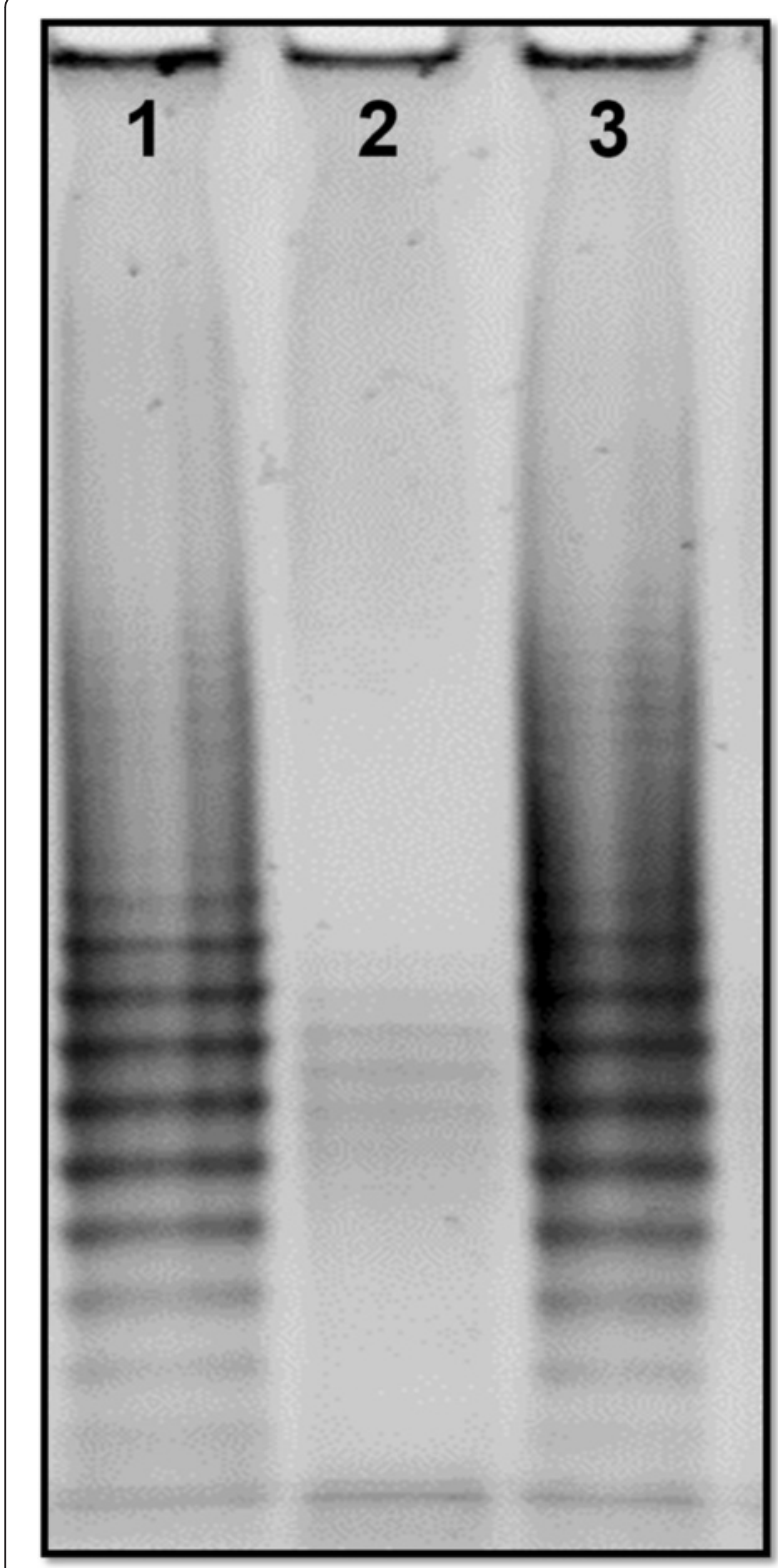

wt waal- waalc

Fig. 2 LPS pattern on a $12 \%$ PAGE gel. Compared to the wt (1), 0 antigen ladder was missing in the waal $^{-}$(2) and in the complemented strain waal $^{c}(3)$, the $\mathrm{O}$ antigen ladder was restored

culture, then became significantly higher at $6 \mathrm{~h}$ and/or $12 \mathrm{~h}$ (One way ANOVA, $P<0.05$, post hoc with Tukey's multiple comparisons test). The complementation restored the growth to normal pattern, as the waaL $L^{c}$ cells showed similar growth rate as the wt cells (Table 1). Like the pattern in the gut, the mutant cells were less competitive in the coculture assay as well. Taken together, the lack of O-PS did not affect bacterial proliferation when growing alone in culture (Fig. 3) but did compromise the competency to

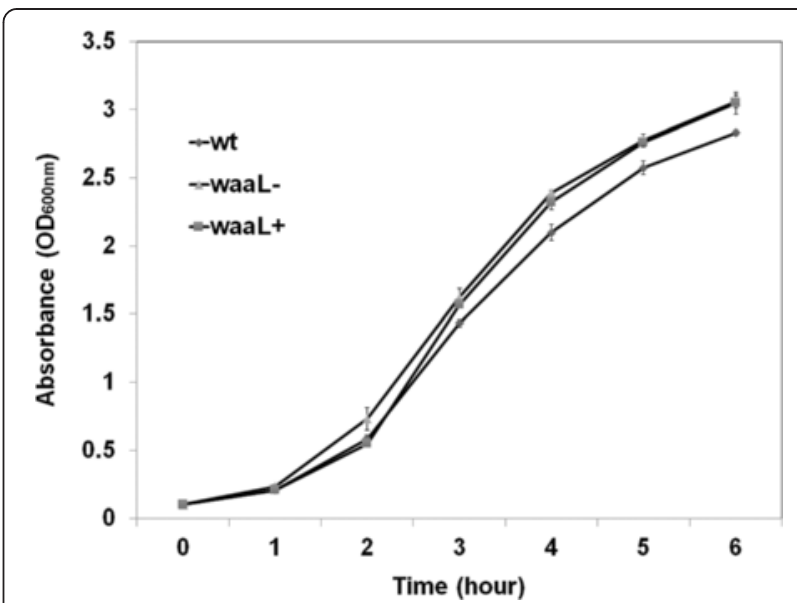

Fig. 3 Growth pattern of the wt, waaL ${ }^{-}$and waal ${ }^{c}$. Bacteria were cultured in the LB medium. The absorbance of $\mathrm{OD}_{600 \mathrm{~nm}}$ was measured hourly. The data were presented with mean \pm SD of absorbance from three replicates

compete with other cells both in vitro (Table 1) and in vivo (Fig. 4).

\section{The mutant bacteria were vulnerable in the presence of} blood in vitro

Enterobacter thrived in the blood fed gut. The LPS deficiency may render mutants more vulnerable to the innate immune factors in the vertebrate blood. To examine the effect of blood on the mutant strain, the cells were incubated in heparinized mouse blood for 1 and $3 \mathrm{~h}$, respectively. Cells were then spread on LB plates with appropriate dilutions. The cell viability was measured by CFU on the plates. At $1 \mathrm{~h}$ post blood incubation, the CFU increased 2.6 \pm 0.5 times in wt, $2.3 \pm 0.2$ times in $w a a L^{c}$, and $2.0 \pm 0.4$ times in $w a a L^{-}$, compared to the CFU at initial time point $(0 \mathrm{~h})$, there was no significant difference among them (One way ANOVA, $P>0.05$ ). However, at $3 \mathrm{~h}$ post incubation, the CFU of wt and waaL $L^{c}$ increased $13.4 \pm 1.7$ and $8.8 \pm 1.4$ times, respectively, while the CFU increased only 7.5 \pm 0.9 times in wal $^{-}$(One way ANOVA, $\left.P<0.05\right)$. The viability of $w_{a a L^{-}}$was lower than that of the wt, suggesting that O-PS structure may be involved in the protection from harmful factors in the blood. The CFU of $w a a L^{c}$ were more than that of $\mathrm{waaL}^{-}$, but the difference was not significant $(P>0.05)$.

The waaL mutants were more sensitive to $\mathrm{H}_{2} \mathrm{O}_{2}$

The waL $^{-}$mutant was obtained by screening the mutant library with paraquat, an oxidative stressor (see Methods). In line with reports in the literature, the waaL mutants in Edwardsiella tarda [32], Pseudomonas aeruginosa and Erwinia amylovora [33] showed higher sensitivity to oxidative stress. Therefore the effect of waaL mutation on the vulnerability to oxidative stress was examined. The 


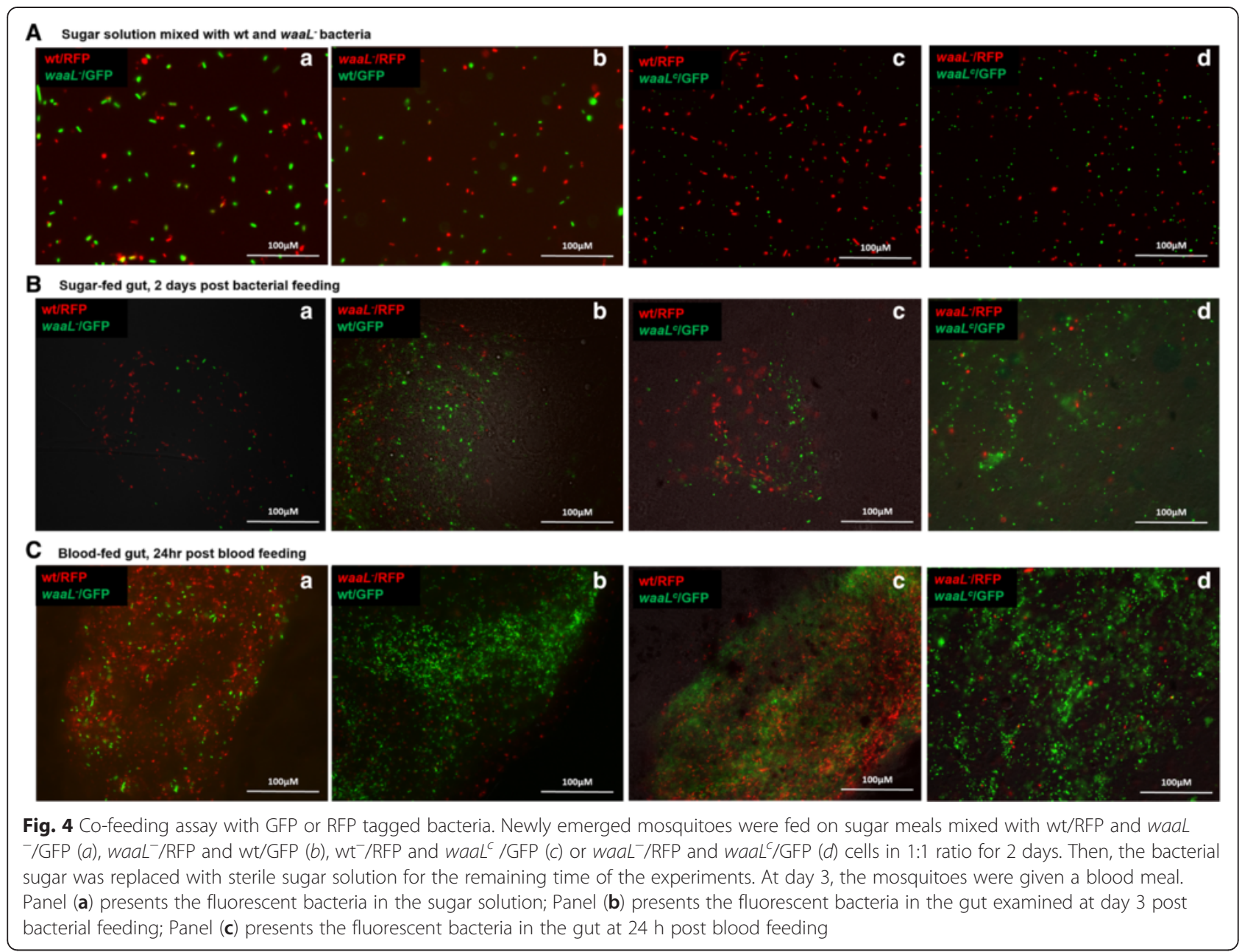

sensitivity to $\mathrm{H}_{2} \mathrm{O}_{2}$ was compared for the wt, waaL and $w a a L^{c}$. The cells were exposed to $20 \mathrm{mM} \mathrm{H}_{2} \mathrm{O}_{2}$ or blank control for $30 \mathrm{~min}$, respectively, and then were spread on LB plates. As shown in Table 2, the waaL had a lower survival rate (39\%) when compared with the wt $(79 \%$, One-way ANOVA, $P<0.01$, Dunnett's comparison versus the wt, $P<0.05)$. The complementation of $w a a L^{c}$ partially restored the protection, the survival rate (59\%) was close to that of the wt (Dunnett's comparison, $P>0.05)$.

Table 1 The growth ratio of wt/mutant in the co-culture

\begin{tabular}{|c|c|c|c|}
\hline \multirow[t]{2}{*}{ Co-culture } & \multicolumn{3}{|c|}{ Ratio $(\text { mean } \pm S D)^{a}$} \\
\hline & $\mathrm{Oh}$ & $6 \mathrm{~h}$ & $12 \mathrm{~h}$ \\
\hline wt (GFP)/ waal- (RFP) & $1.19 \pm 0.07^{a}$ & $3.64 \pm 0.92^{a b}$ & $5.24 \pm 1.77^{b}$ \\
\hline waal $^{c}(\mathrm{RFP}) /$ waal $^{-}$(GFP) & $1.34 \pm 0.01^{\mathrm{a}}$ & $4.4 \pm 1.38^{b}$ & $6.85 \pm 0.64^{b}$ \\
\hline wt (GFP)/ waal ${ }^{c}$ (RFP) & $0.98 \pm 0.12^{a}$ & $1.29 \pm 0.07^{\mathrm{a}}$ & $1.8 \pm 0.65^{a}$ \\
\hline
\end{tabular}

${ }^{a}$ The numbers of fluorescent cell in a co-culture were numerated at 0,6 and $12 \mathrm{~h}$ and ratios were calculated. The ratios in each co-culture were analyzed using one way ANOVA. Tukey test was used as post hoc comparison. Significant difference was indicated by different letters.
The mutant-fed mosquitoes were more susceptible to rodent malaria

Some species of Enterobacter have been shown to be involved in the mosquito immunity against malaria $[6,16$, 24]. To determine if the waaL mutation affect mosquito susceptibility to rodent malaria Plasmodium berghei, malarial infection patterns were compared in the mosquitoes fed on the wt and waaL $L^{-}$mutants. Mosquitoes were set as three cohorts. One cohort was fed on the regular sugar as a control. The other two cohorts were fed on sugar

Table 2 Sensitivity to hydrogen peroxide

\begin{tabular}{lccr}
\hline & \multicolumn{3}{c}{ Cell counts and survival rates (mean \pm SD) } \\
\cline { 2 - 4 } & wt & waaL $^{-}$ & waaL $^{c}$ \\
\hline $\mathrm{H}_{2} \mathrm{O}_{2}$ absent & $856.3 \pm 51.4$ & $685.0 \pm 91.0$ & $758.0 \pm 69.4$ \\
$\mathrm{H}_{2} \mathrm{O}_{2}$ present & $674.3 \pm 111.6$ & $269.0 \pm 91.9$ & $443.7 \pm 19.1$ \\
Survival rate & $0.79 \pm 0.16$ & $0.39 \pm 0.08$ & $0.59 \pm 0.06$
\end{tabular}

The cell counts post exposure to $20 \mathrm{mM} \mathrm{H}_{2} \mathrm{O}_{2}$ were obtained from three replicates. The survival rates were analyzed by one way ANOVA $(P<0.01)$. Dunnett's method was used for comparisons, waaL ${ }^{-}$vs wt, $P<0.05$; waa $L^{c}$ vs wt, $P>0.05$ 
supplemented with the wt bacteria and the waaL $L^{-}$bacteria, respectively. The three cohorts were then fed on the same set of mice infected with $P$. berghei. The malaria infection was assessed by the prevalence and the oocyst number per gut. As shown in Fig. 5, the control mosquitoes that were fed on regular sugar showed prevalence of $80 \%$ and average parasite load of 83.5 oocyst/gut. The wt-fed mosquitoes showed reduced prevalence of infection (52.5\%) and less parasite load (mean = 33 oocyst/gut), while the mutant-fed mosquitoes had higher infection (mean $=184.9$ oocyst $/$ gut, $87.5 \%$ of prevalence). The prevalence of infection between the wt and mutant was significantly different $\left(\chi^{2}\right.$ test, $P<0.01)$, and the oocyst load among three cohorts was statistically different as well (Kruskal-Wallis ANOVA on ranks, $P<0.01$, Dunn's all pairwise multiple comparison procedures, $P<0.05$ ). In a replicate experiment, the same pattern was observed (data not shown).

\section{Discussion}

Transposon mediated mutagenesis has been widely used in bacterial gene function study [34-36]. In this study, we generated a Tn5 mediated mutant library of Enterobacter sp. Ag1. The waaL ${ }^{-}$mutant was characterized. The disruption of the waaL abolished the attachment of O-PS to the outer core of LPS, which resulted in the O-PS deficiency. The LPS in the wt bacteria displayed a ladder of O-PS repeat units on a PAGE gel, a typical pattern of smooth type of LPS. The banding pattern of O-PS repeats was absent in the waaL $^{-}$mutant, the pattern was restored by complementation as expected (Fig. 2), indicating the requirement of waaL in the O-PS attachment during LPS synthesis in Enterobacter sp. Ag1. There are two types of

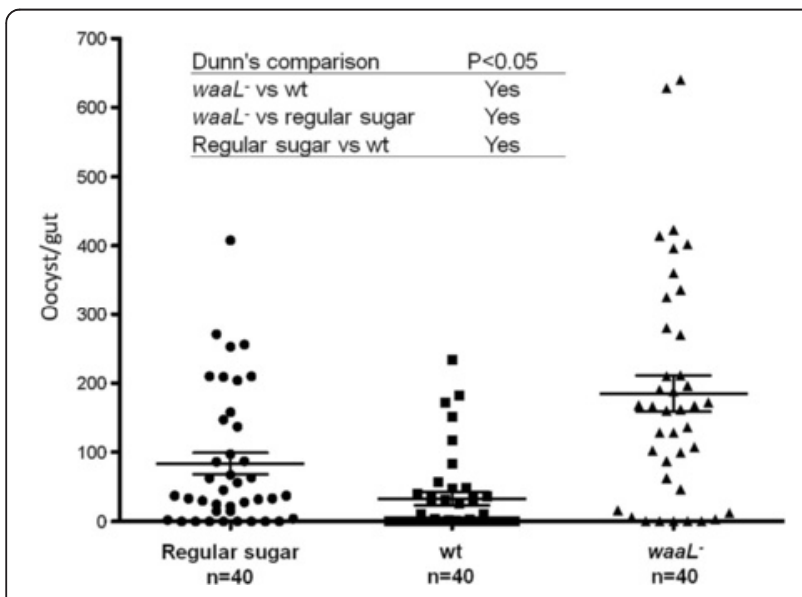

Fig. 5 The effect of Enterobacter strains on mosquito susceptibility to malaria $P$. berghei infection. The parasite loads of each individual in the three cohorts were presented. The mean \pm SD of oocysts was indicated by the horizontal line. The parasite loads were significantly different among the three cohorts (ANOVA on rank, $P<0.01$ ). Dunn's comparisons between the cohorts were presented in the insert panel.
LPS in Gram negative cells, the smooth type that O-PS is present and the rough type that O-PS is absent [37].

The LPS defect appeared not to interfere with the growth when the mutant was alone in the LB culture. The waaL grew in a rate similar to the wt and complemented bacteria (Fig. 3). However, when growing together with the wt cells in the co-culture assay, the mutant cells grew at a slower rate (Table 1). A similar pattern was observed in vivo. In the co-feeding assay, after being ingested via sugar meals, the wt bacteria steadily populated in the gut, while the waaL $L^{-}$bacteria were less prevalent in both sugar-fed and blood-fed guts (Fig. 4). These findings suggest that the absence of O-antigen compromised the competency of proliferation in co-culture in vitro and co-feeding assays in vivo, although the conditions in LB culture and in the gut are quite different. Interestingly, the $\mathrm{O}$-antigen has been associated with the social gliding mobility (cells move as groups) in Myxococcus xanthus [38] and social swarming mobility in Pectobactrium atrosepticum [39]. In a community setting, cell-cell interactions may be essential for bacterial growth. The lack of O-antigen in the mutants made it less competent in proliferation in both co-culture and cofeeding assays (Table 1, Fig. 4), suggesting the $\mathrm{O}$-antigen may be involved in cell-cell interactions in a microbial community setting.

In the blood-fed gut, more complicated situations could happen. The mammalian blood contains hostile immune factors such as antibodies and complement. It has been documented that human complement was active in the gut for 1 hour after ingestion [40], the Aedes aegypti mosquitoes produced complement system inhibitors to protect gut epithelial cells from possible injury by the complement [41]. These mammalian immune factors may adversely affect bacteria in the gut. It has been shown that serum antibodies were able to mediate complement-dependent killing of various bacteria [42, 43]. In some cases, the $\mathrm{O}$-antigen could confer bacteria resistance to host immune defenses especially to complement protein deposition and complement lytic activity [44, 45]. In Bordetella parapertussis $\mathrm{O}$ antigen dependent protection against complement-mediated control and clearance was necessary for the bacteria to efficiently colonize the lower respiratory tract [45]. In our case, the $w_{a a L^{-}}$was less viable at $3 \mathrm{~h}$ post incubation with the blood in vitro. It is conceivable that mammalian host immune factors in a blood meal may contribute to the competition defect in the blood-fed gut in the co-feeding assay. Taken together, the waaL $L^{-}$defect in competition either in vitro or in vivo may associate with different mechanisms in different scenarios. Further studies are necessary to characterize these context dependent mechanisms. In the squid-Vibrio symbiotic system, the LPS structure, especially O-antigen, is required for Vibrio fischeri to colonize the squid light organ [46]. Similarly, the O-antigendeficiency in Salmonella enterica serovar typhimurium 
compromised its colonization in the Peyer's patches and liver of mice [47]. In Rhizobium tropici CIAT899 LPS defects impaired maize rhizosphere and root colonization [48]. Recently, it was shown that a phosphatase mediated lipid A modulation was required for the periodontal colonization of Porphyromonas gingivalis in a rabbit model [49]. These data exemplified solid connections between LPS structure and host colonization in various circumstances.

The $\mathrm{waal}^{-}$mutant was selected by paraquat screening. It was sensitive to $\mathrm{H}_{2} \mathrm{O}_{2}$ as well (Table 2). The results were reminiscent of the phenotypes of waaL mutants of Erwinia amylovora, Pseudomonas aeruginosa [33] and Edwardsiella tarda [32]. The waaL mutation in these species resulted in higher sensitivity to $\mathrm{H}_{2} \mathrm{O}_{2}$. At the present, it is not clear how the LPS deficiency impairs the defense against oxidative stress in the $w a a L^{-}$mutant. A possible explanation is that defective LPS may render the outer membrane less protective, which may increase permeability to oxidants. The O-antigen dependent protection against oxidative stress may be of ecological significance for Enterobacter to thrive in the blood-fed gut [7]. Blood feeding makes mosquito gut a stressful environment due to the elevated oxidative stress that is associated with the hemoglobin digestion and metabolism [50]. The bacteria and ingested blood are confined by a transient peritrophic matrix that is produced by mosquito gut cells to spatially separate the blood bolus from the gut epithelia [51]. Therefore, the gut bacteria are directly exposed to the oxidative stress derived from blood digestion. We have shown that blood feeding shifts microbial structure in the mosquito gut by reducing the taxonomic diversity and increasing the abundance of bacteria in the family Enterobacteriaceae [7]. In the bloodfed gut, the LPS dependent stress defense may be one of the protections for bacteria to thrive in the stressful environment. The impaired defense against oxidative stress in the waa $L^{-}$may contribute to the competition defect in the blood-fed gut. In maintaining a healthy host microbial symbiosis, redox homeostasis plays a pivotal role in cross-talks among different parties in a host associated metagenomic ecosystem [52]. The findings in the current study suggest that an intact LPS structure is critical for Enterobacter to reside stably in the mosquito gut by several different means possibly from protections to cell-cell interactions.

The gut microbial community plays an essential role in maintaining a basal immunity in mosquitoes [11, 22] . In the family Enterobacteriaceae, some strains of Enterobacter [16, 24, 53] and Serratia [54] have been shown to be influential in malaria susceptibility. In line with those observations, wt-fed mosquitoes had lower $P$. berghei infection, while the waa $L^{-}$-fed mosquitoes showed higher infection (Fig. 5). The data suggest that the wt Enterobacter could populate the gut well and promoted stronger immunity against malaria. This effect was compromised in the mutants, likely due to the less competitiveness in the gut community (Fig. 4). Alternatively, LPS may be immunogenic to prime basal immunity in the gut. It is worth noticing that the waaL gene of Aeromonas hydrophila was required for stimulation of melanization in the insect, Tenebrio molitor [55], and in Maduca sexta the LPS with O-antigen was more potent than the lipid A and rough mutants of LPS in activation of antimicrobial gene expression [56], although LPS does not activate Drosophila immune responses in adults $[57,58]$. Further study is required to determine if LPS is involved in the activation of antimalaria immunity. The availability of LPS deficient mutants enables one to study the roles of LPS structure of Enterobacter in the symbiotic relationship with the mosquito host. Further investigation is underway to characterize the dynamics of Enterobacter in the mosquito gut and the impact on the mosquito traits, such as fecundity and gut local immunity.

\section{Conclusions}

In the mosquito gut, Enterobacter is a predominant resident in the microbial community. Lack of the $\mathrm{O}$ antigen structure in LPS of Enterobacter compromised its effective growth in co-culture and reduced its prevalence in the cofeeding assays. In addition, O-antigen deficiency increased the sensitivity to oxidative stress. The findings suggest intact LPS is required for the bacteria to steadily stay in the gut.

\section{Abbreviations \\ LPS: Lipopolysaccharide; CFU: Colony forming unit; GFP: Green fluorescent protein; RFP: Red fluorescent protein; KAN: Kanamycin.}

\section{Competing interests}

The authors declare that they have no competing interests.

\section{Authors' contributions}

JX conceived of the study and participated in its design and coordination. WY generated the mutant library, screened the mutants and generated complemented strain, and tagged bacteria fluorescently. DP JJ, PK characterized the phenotypes of mutants, co-culture and co-feeding assays. $J$ J conducted experiments of malaria infection. AU was a BRIDGES student, he received the training on the project and participated in the gut dissection and fluorescent bacterial imaging. JX, DP and JJ wrote the manuscripts. All authors read and agreed the final version of the manuscript.

\section{Acknowledgements}

This work was supported by the grants SC2GM092789 and SC1GM109367 from the National Institutes of Health, and the grant DMS-1222592 from National Science Foundation. AU was an undergraduate research scholar supported by the $\mathrm{NIH}$ Bridges program.

Received: 7 July 2015 Accepted: 17 August 2015

Published online: 27 August 2015

\section{References}

1. Chavshin AR, Oshaghi MA, Vatandoost H, Pourmand MR, Raeisi A, Enayati $A A$, et al. Identification of bacterial microflora in the midgut of the larvae and adult of wild caught Anopheles stephensi: a step toward finding suitable paratransgenesis candidates. Acta Trop. 2012;121(2):129-34. 
2. Straif SC, Mbogo CN, Toure AM, Walker ED, Kaufman M, Toure YT, et al. Midgut bacteria in Anopheles gambiae and An. funestus (Diptera: Culicidae) from Kenya and Mali. J Med Entomol. 1998;35(3):222-6.

3. Rani A, Sharma A, Rajagopal R, Adak T, Bhatnagar RK. Bacterial diversity analysis of larvae and adult midgut microflora using culture-dependent and culture-independent methods in lab-reared and field-collected Anopheles stephensi-an Asian malarial vector. BMC Microbiol. 2009;9:96.

4. Terenius O, Lindh JM, Eriksson-Gonzales K, Bussiere L, Laugen AT, Bergquist $\mathrm{H}$, et al. Midgut bacterial dynamics in Aedes aegypti. FEMS Microbiol Ecol. 2012;80(3):556-65.

5. Minard G, Mavingui P, Moro CV. Diversity and function of bacterial microbiota in the mosquito holobiont. Parasit Vectors. 2013;6:146.

6. Boissiere A, Tchioffo MT, Bachar D, Abate L, Marie A, Nsango SE, et al. Midgut microbiota of the malaria mosquito vector Anopheles gambiae and interactions with Plasmodium falciparum infection. PLoS Pathog. 2012;8(5), e1002742.

7. Wang Y, Gilbreath 3rd TM, Kukutla P, Yan G, Xu J. Dynamic gut microbiome across life history of the malaria mosquito Anopheles gambiae in Kenya. PLoS One. 2011;6(9), e24767.

8. Chavshin AR, Oshaghi MA, Vatandoost H, Pourmand MR, Raeisi A, Terenius O. Isolation and identification of culturable bacteria from wild Anopheles culicifacies, a first step in a paratransgenesis approach. Parasit Vectors. 2014;7:419.

9. Pumpuni CB, Demaio J, Kent M, Davis JR, Beier JC. Bacterial population dynamics in three anopheline species: the impact on Plasmodium sporogonic development. Am J Trop Med Hyg. 1996;54(2):214-8.

10. Osei-Poku J, Mbogo CM, Palmer WJ, Jiggins FM. Deep sequencing reveals extensive variation in the gut microbiota of wild mosquitoes from Kenya. Mol Ecol. 2012;21(20):5138-50.

11. Dong Y, Manfredini F, Dimopoulos G. Implication of the mosquito midgut microbiota in the defense against malaria parasites. PLoS Pathog. 2009;5(5), e1000423.

12. Lindh JM, Borg-Karlson AK, Faye I. Transstadial and horizontal transfer of bacteria within a colony of Anopheles gambiae (Diptera: Culicidae) and oviposition response to bacteria-containing water. Acta Trop. 2008;107(3):242-50.

13. Noden BH, Vaughan JA, Pumpuni CB, Beier JC. Mosquito ingestion of antibodies against mosquito midgut microbiota improves conversion of ookinetes to oocysts for Plasmodium falciparum, but not $P$. yoelii. Parasitol Int. 2011;60(4):440-6.

14. Sharma A, Dhayal D, Singh OP, Adak T, Bhatnagar RK. Gut microbes influence fitness and malaria transmission potential of Asian malaria vector Anopheles stephensi. Acta Trop. 2013;128(1):41-7.

15. Gusmao DS, Santos AV, Marini DC, Bacci Jr M, Berbert-Molina MA, Lemos FJ. Culture-dependent and culture-independent characterization of microorganisms associated with Aedes aegypti (Diptera: Culicidae) (L.) and dynamics of bacterial colonization in the midgut. Acta Trop. 2010;115(3):275-81

16. Gonzalez-Ceron L, Santillan F, Rodriguez MH, Mendez D, Hernandez-Avila JE. Bacteria in midguts of field-collected Anopheles albimanus block Plasmodium vivax sporogonic development. J Med Entomol. 2003;40(3):371-4

17. Mitraka E, Stathopoulos S, Siden-Kiamos I, Christophides GK, Louis C. Asaia accelerates larval development of Anopheles gambiae. Pathog Glob Health. 2013;107(6):305-11.

18. Chouaia B, Rossi P, Epis S, Mosca M, Ricci I, Damiani C, et al. Delayed larval development in Anopheles mosquitoes deprived of Asaia bacterial symbionts. BMC Microbiol. 2012;12 Suppl 1:S2

19. Capone A, Ricci I, Damiani C, Mosca M, Rossi P, Scuppa P, et al. Interactions between Asaia, Plasmodium and Anopheles: new insights into mosquito symbiosis and implications in malaria symbiotic control. Parasit Vectors. 2013;6(1):182

20. Tchioffo MT, Boissiere A, Churcher TS, Abate L, Gimonneau G, Nsango SE, et al. Modulation of malaria infection in Anopheles gambiae mosquitoes exposed to natural midgut bacteria. PLoS One. 2013;8(12), e81663.

21. Coon KL, Vogel KJ, Brown MR, Strand MR. Mosquitoes rely on their gut microbiota for development. Mol Ecol. 2014;23(11):2727-39.

22. Bahia AC, Dong Y, Blumberg BJ, Mlambo G, Tripathi A, BenMarzouk-Hidalgo OJ, et al. Exploring Anopheles gut bacteria for Plasmodium blocking activity. Environ Microbiol. 2014;16(9):2980-94

23. Favia G, Ricci I, Damiani C, Raddadi N, Crotti E, Marzorati M, et al. Bacteria of the genus Asaia stably associate with Anopheles stephensi, an Asian malarial mosquito vector. Proc Natl Acad Sci U S A. 2007;104(21):9047-51.
24. Eappen AG, Smith RC, Jacobs-Lorena M. Enterobacter-activated mosquito immune responses to Plasmodium involve activation of SRPN6 in Anopheles stephensi. PLoS One. 2013;8(5), e62937.

25. Jiang J, Alvarez C, Kukutla P, Yu W, Xu J. Draft genome sequences of Enterobacter sp. isolate Ag1 from the midgut of the malaria mosquito Anopheles gambiae. J Bacteriol. 2012;194(19):5481.

26. Fire A, Harrison SW, Dixon D. A modular set of lacZ fusion vectors for studying gene expression in Caenorhabditis elegans. Gene. 1990;93(2):189-98.

27. Moncayo AC, Lerdthusnee K, Leon R, Robich RM, Romoser WS. Meconial peritrophic matrix structure, formation, and meconial degeneration in mosquito pupae/pharate adults: histological and ultrastructural aspects. J Med Entomol. 2005;42(6):939-44.

28. Moll RM, Romoser WS, Modrzakowski MC, Moncayo AC, Lerdthusnee K. Meconial peritrophic membranes and the fate of midgut bacteria during mosquito (Diptera: Culicidae) metamorphosis. J Med Entomol. 2001;38(1):29-32.

29. Romoser WS, Moll RM, Moncayo AC, Lerdthusnee K. The occurrence and fate of the meconium and meconial peritrophic membranes in pupal and adult mosquitoes (Diptera: Culicidae). J Med Entomol. 2000;37(6):893-6.

30. Raetz CR, Whitfield C. Lipopolysaccharide endotoxins. Annu Rev Biochem. 2002;71:635-700

31. Heinrichs DE, Monteiro MA, Perry MB, Whitfield C. The assembly system for the lipopolysaccharide R2 core-type of Escherichia coli is a hybrid of those found in Escherichia coli K-12 and Salmonella enterica. Structure and function of the R2 WaaK and WaaL homologs. J Biol Chem. 1998;273(15):8849-59.

32. Xu L, Wang Q, Xiao J, Liu Q, Wang X, Chen T, et al. Characterization of Edwardsiella tarda waaL: roles in lipopolysaccharide biosynthesis, stress adaptation, and virulence toward fish. Arch Microbiol. 2010;192(12):1039-47.

33. Berry MC, McGhee GC, Zhao Y, Sundin GW. Effect of a waaL mutation on lipopolysaccharide composition, oxidative stress survival, and virulence in Erwinia amylovora. FEMS Microbiol Lett. 2009;291(1):80-7.

34. Saroj SD, Clemmer KM, Bonomo RA, Rather PN. Novel mechanism for fluoroquinolone resistance in Acinetobacter baumannii. Antimicrob Agents Chemother. 2012:56(9):4955-7.

35. Xu J, Deng P, Showmaker KC, Wang H, Baird SM, Lu SE. The pqqC gene is essential for antifungal activity of Pseudomonas kilonensis JX22 against Fusarium oxysporum f. sp. Iycopersici. FEMS Microbiol Lett. 2014;353(2):98-105.

36. Cheong H, Kim CY, Jeon JS, Lee BM, Sun Moon J, Hwang I. Xanthomonas oryzae pv. oryzae type III effector XopN targets OsVOZ2 and a putative thiamine synthase as a virulence factor in rice. PLoS One. 2013;8(9):e73346.

37. Lerouge I, Vanderleyden J. O-antigen structural variation: mechanisms and possible roles in animal/plant-microbe interactions. FEMS Microbiol Rev. 2002;26(1):17-47

38. Bowden MG, Kaplan HB. The Myxococcus xanthus lipopolysaccharide O-antigen is required for social motility and multicellular development. Mol Microbiol. 1998;30(2):275-84.

39. Bowden SD, Hale N, Chung JC, Hodgkinson JT, Spring DR, Welch M. Surface swarming motility by Pectobacterium atrosepticum is a latent phenotype that requires $\mathrm{O}$ antigen and is regulated by quorum sensing. Microbiology. 2013;159(Pt 11):2375-85.

40. Simon N, Lasonder E, Scheuermayer M, Kuehn A, Tews S, Fischer R, et al. Malaria parasites co-opt human factor $\mathrm{H}$ to prevent complement-mediated lysis in the mosquito midgut. Cell Host Microbe. 2013;13(1):29-41.

41. Barros VC, Assumpcao JG, Cadete AM, Santos VC, Cavalcante RR, Araujo RN, et al. The role of salivary and intestinal complement system inhibitors in the midgut protection of triatomines and mosquitoes. PLoS One. 2009;4(6), e6047.

42. Tichaczek-Goska D, Witkowska D, Cisowska A, Jankowski S, Hendrich AB. The bactericidal activity of normal human serum against Enterobacteriaceae rods with lipopolysaccharides possessing O-antigens composed of mannan. Adv Clin Exp Med. 2012;21(3):289-99.

43. Frank MM, Joiner $\mathrm{K}$, Hammer $\mathrm{C}$. The function of antibody and complement in the lysis of bacteria. Rev Infect Dis. 1987;9 Suppl 5:S537-45.

44. Clay CD, Soni S, Gunn JS, Schlesinger LS. Evasion of complement-mediated lysis and complement C3 deposition are regulated by Francisella tularensis lipopolysaccharide O antigen. J Immunol. 2008;181(8):5568-78.

45. Goebel EM, Wolfe DN, Elder K, Stibitz S, Harvill ET. O antigen protects Bordetella parapertussis from complement. Infect Immun. 2008;76(4):1774-80. 
46. Post DM, Yu L, Krasity BC, Choudhury B, Mandel MJ, Brennan CA, et al. O-antigen and core carbohydrate of Vibrio fischeri lipopolysaccharide: composition and analysis of their role in Euprymna scolopes light organ colonization. J Biol Chem. 2012;287(11):8515-30.

47. Kong Q, Yang J, Liu Q, Alamuri P, Roland KL, Curtiss 3rd R. Effect of deletion of genes involved in lipopolysaccharide core and O-antigen synthesis on virulence and immunogenicity of Salmonella enterica serovar typhimurium. Infect Immun. 2011;79(10):4227-39.

48. Ormeno-Orrillo E, Rosenblueth M, Luyten E, Vanderleyden J, Martinez-Romero E. Mutations in lipopolysaccharide biosynthetic genes impair maize rhizosphere and root colonization of Rhizobium tropici CIAT899. Environ Microbiol. 2008; 10(5):1271-84.

49. Zenobia C, Hasturk H, Nguyen D, Van Dyke TE, Kantarci A, Darveau RP. Porphyromonas gingivalis Lipid A Phosphatase Activity Is Critical for Colonization and Increasing the Commensal Load in the Rabbit Ligature Model. Infect Immun. 2014;82(2):650-9.

50. Graca-Souza AV, Maya-Monteiro C, Paiva-Silva GO, Braz GR, Paes MC, Sorgine $\mathrm{MH}$, et al. Adaptations against heme toxicity in blood-feeding arthropods. Insect Biochem Mol Biol. 2006;36(4):322-35.

51. Lehane MJ. Peritrophic matrix structure and function. Annu Rev Entomol. 1997:42:525-50.

52. Mone $\mathrm{Y}$, Monnin D, Kremer N. The oxidative environment: a mediator of interspecies communication that drives symbiosis evolution. Proc Biol Sci. 2014;281(1785):20133112.

53. Cirimotich CM, Dong Y, Clayton AM, Sandiford SL, Souza-Neto JA, Mulenga $M$, et al. Natural microbe-mediated refractoriness to Plasmodium infection in Anopheles gambiae. Science. 2011;332(6031):855-8.

54. Bando H, Okado K, Guelbeogo WM, Badolo A, Aonuma H, Nelson B, et al. Intra-specific diversity of Serratia marcescens in Anopheles mosquito midgut defines Plasmodium transmission capacity. Sci Rep. 2013;3:1641.

55. Noonin C, Jiravanichpaisal P, Soderhall I, Merino S, Tomas JM, Soderhall K. Melanization and pathogenicity in the insect, Tenebrio molitor, and the crustacean, Pacifastacus leniusculus, by Aeromonas hydrophila AH-3. PLOS One. 2010;5(12), e15728.

56. Rao XJ, Yu XQ. Lipoteichoic acid and lipopolysaccharide can activate antimicrobial peptide expression in the tobacco hornworm Manduca sexta. Dev Comp Immunol. 2010;34(10):1119-28.

57. Leulier F, Parquet C, Pili-Floury S, Ryu JH, Caroff M, Lee WJ, et al. The Drosophila immune system detects bacteria through specific peptidoglycan recognition. Nat Immunol. 2003;4(5):478-84.

58. Kaneko T, Goldman WE, Mellroth P, Steiner H, Fukase K, Kusumoto S, et al. Monomeric and polymeric gram-negative peptidoglycan but not purified LPS stimulate the Drosophila IMD pathway. Immunity. 2004;20(5):637-49.

\section{Submit your next manuscript to BioMed Central and take full advantage of:}

- Convenient online submission

- Thorough peer review

- No space constraints or color figure charges

- Immediate publication on acceptance

- Inclusion in PubMed, CAS, Scopus and Google Scholar

- Research which is freely available for redistribution 